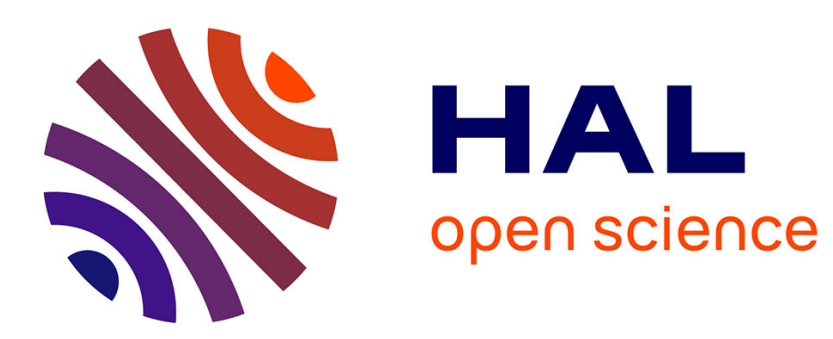

\title{
The Fabrication of Circular Cross Section Waveguide in Two Dimensions with a Dynamical Slit
}

Y. Zhang, G. Cheng, G. Huo, Y. Wang, W. Zhao, Cyril Mauclair, Razvan

Stoian, R. Hui

\section{- To cite this version:}

Y. Zhang, G. Cheng, G. Huo, Y. Wang, W. Zhao, et al.. The Fabrication of Circular Cross Section Waveguide in Two Dimensions with a Dynamical Slit. Laser Physics, 2009, 19 (12), pp.2236-2241. 10.1134/S1054660X0923008X 。 ujm-00447363

\section{HAL Id: ujm-00447363}

\section{https://hal-ujm.archives-ouvertes.fr/ujm-00447363}

Submitted on 2 Jun 2010

HAL is a multi-disciplinary open access archive for the deposit and dissemination of scientific research documents, whether they are published or not. The documents may come from teaching and research institutions in France or abroad, or from public or private research centers.
L'archive ouverte pluridisciplinaire HAL, est destinée au dépôt et à la diffusion de documents scientifiques de niveau recherche, publiés ou non, émanant des établissements d'enseignement et de recherche français ou étrangers, des laboratoires publics ou privés. 


\title{
The Fabrication of Circular Cross-Section Waveguide in Two Dimensions with a Dynamical Slit ${ }^{1}$
}

\author{
Y. Zhang ${ }^{a}$, G. Cheng ${ }^{a} *$, G. Huo ${ }^{a}$, Y. Wang ${ }^{a}$, W. Zhao ${ }^{a}$, C. Mauclair ${ }^{b}$, R. Stoian ${ }^{b}$, and R. Hui ${ }^{c}$ \\ ${ }^{a}$ State Key Laboratory of Transient Optics and Photonics, Xi'an Institute of Optics and Precision Mechanics, \\ CAS, Xi'an Shaanxi 710119, China \\ ${ }^{b}$ Laboratoire Hubert Curien, UMR 5516 CNRS, Universite Jean Monnet, 42000 Saint Etienne, Universite de Lyon, \\ 42023 Saint Etienne, France \\ ${ }^{c}$ Department of Electrical Engineering and Computer Science, The University of Kansas, Lawrence, \\ Kansas 66044, USA \\ *e-mail: gcheng@opt.ac.cn \\ Received June 29, 2009; in final form, July 3, 2009; published online October 23, 2009
}

\begin{abstract}
A waveguide with a circular transverse profile can be obtained by using a slit beam shaping method. Applications about optical circuit chips in glass require a circular cross-section waveguide in two dimensions. Here we reported to realize a fabrication of circular cross-section waveguide in two dimensions by rotating the slit corresponding to the tangent line of the arc of the waveguide. The dependence of cross-section on width of the slit was investigated in experiment. The difference of slit width between geometrical optics prediction and experimental result was explained by considering the focal shift in xy directions due to diffraction effects.
\end{abstract}

PACS numbers: 42.82.Et, 42.81.Qb, 42.65. Tg, 42.55.Rz

DOI: $10.1134 / \mathrm{S} 1054660 \mathrm{X} 0923008 \mathrm{X}$

\section{INTRODUCTION}

Femtosecond laser optical waveguide writing has attracted a lot of interest as it continuously demonstrates its capability to achieve complex three-dimensional photonic structures with very simple equipment in rapid prototyping process [1-3]. Longitudinal and transverse writing geometries are possible by adjusting the relative translation between the beam and the sample. In the longitudinal geometry, the waveguides are intrinsically symmetric, however the waveguide dimension is limited by the focal length of the focusing objective [4, 5], and homogeneous longitudinal waveguide writing requires dynamical aberration compensation [6]. The transverse geometry provides a much greater flexibility and allows one to write waveguides or photonic circuits of arbitrary length and complexity. However it has the disadvantage of producing a strong asymmetry in the waveguide crosssection. The waveguide cross-section has a width equal to about twice the beam waist, while it has a dimension in depth of the order of the confocal parameter, which is typically much larger. This problem can be overcome by the use of suitable beam shaping techniques $[7,8]$. Circle cross-section waveguides in silica, polymer and borate glass has been successfully demonstrated in one dimensional writing scheme [9-12]. Nonlinear effect has been considered and achieved deep writing in fused silica with a mechanical slit [13]. Also a two-dimensional deformable mirror to

\footnotetext{
1 The article is published in the original.
}

shape the spatial profile of an ultrafast laser beam was demonstrated to inscribe circular cross-section waveguide in soda-lime silica glass slide [14]. In fact, astigmatic writing beam worse the waveguide crosssection if the sample is translated parallel to the short axis of the astigmatic beam. So a cylindrical telescope or a fixed slit can't achieve two-dimensional circle cross-section waveguide which is normal required in planar light-wave circuit (PLC) chip, such as directional coupler, wavelength-division-multiplexed (WDM), arrayed waveguide grating (AWG) [15]. In order to obtain circle cross-section waveguide in two dimensions, a simple method is to rotate the slit corresponding to the waveguide configuration. More particular, the long axis of the astigmatic writing beam keeps parallel to the tangent line of the waveguide arc. In this article, a mechanical slit fixed on a computercontrolled $360^{\circ}$ rotation stage is used as a dynamical slit to realize $2 \mathrm{D}$ circle cross-section waveguide.

\section{THEORY}

Since the first report on realization circular crosssection waveguide writing with astigmatic laser beam [7], it is the key consideration to understand how to calculate the aspect ratio of truncated Gaussian beam or elliptical Gaussian beam. Geometry optics has been employed based on a condition that the beam waist at one direction is set to equal to the confocal parameter [7, 11], and an aspect ratio of about 3 has been obtained. However it seams to be underestimated, low 
(a)

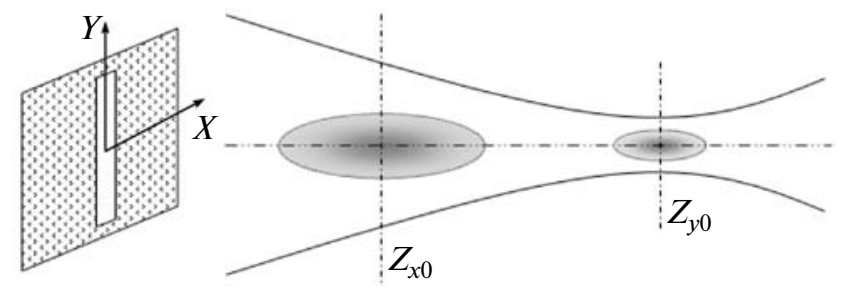

Focal shift, mm

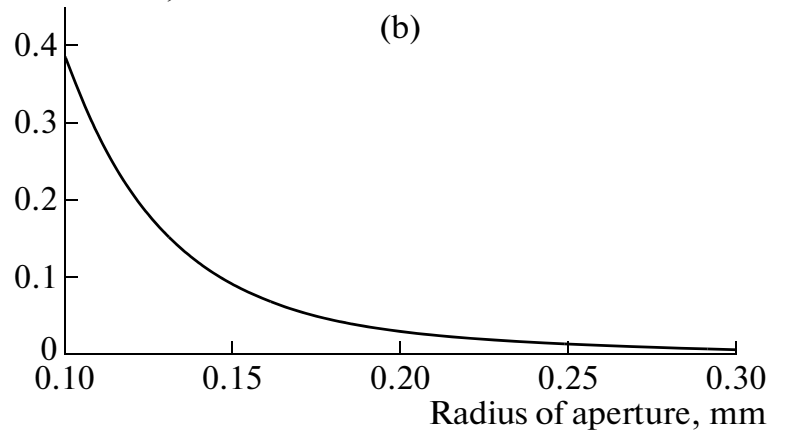

Fig. 1. Focal positions of a focused Gaussian beam after a slit (a). Due to Fresnel numbers are different for two orthogral directions, focal shift at $\mathrm{x}$ direction is more bigger than $y$ direction. As a results, the real focus of focused Gaussian beam after slit will locate between $Z_{x 0}$ and $Z_{y 0}$. (b) Shows the dependence of focal shift on radius of aperture.

repetition rate laser experimental results (no strong thermal effect) is bigger than the theoretic prediction. Then diffraction method has been proposed [16]. The writing of circular cross-section waveguide has been demonstrated in simulation and experiment with a 12 aspect ratio, which is bigger than most experimental results so far $[17,18]$ if neglecting the material difference and nonlinear effects during writing a waveguide with femtosecond laser. In spite of this, diffraction theory is an accurate method to this problem, just need special algorithm to get high precise solution. Here we tried to improve geometrical models by using a truncated Gaussian beam model. And find that geometrical optics can't hold any more due to intense diffraction. A simple model is proposed to explain how diffraction affects the focus position and confocal parameter.

In an attempt to improve the model which based on geometry optics, we compare the results under three kinds of beam parameters, plane wave in $x$ and $y$ directions, Gaussian distribution in $x$ and $y$ directions, and Gaussian beam in $y$ direction and plane wave in $x$ directions. The position of the slit is shown in Fig. 1. Beam parameters and focusing objective parameters are as following: the diameter of laser beam is $6 \mathrm{~mm}$, the NA of focusing objective is 0.55 , the effective focal length of objective is $4 \mathrm{~mm}$, and the wavelength of the writing laser is $800 \mathrm{~nm}$. Here we used $50 \%$ maximum as the radius of beam waist considering that the definition of Rayleigh length is the distance along the propagation direction of a beam from the waist to the place where the area of the cross section is doubled, that is, intensity is half of the maximum. For plane wave the beam waist is $0.84 \lambda / N A$, and the beam waist of Gaussian beam goes to $\omega_{Y}=1.09 \lambda / N A$ when truncated ratio goes to infinity, and $\omega_{X}=1.03 \lambda / N A$ [19]. The laser intensity distribution near the laser focus decides the cross-section configuration of waveguide, the principle to realize a circular cross-section waveguide is to keep the bigger beam waist between two beam waists at orthogonal directions ( $X$ direction beam waist in this paper) equal to the confocal length (twice Rayleigh length). Here Rayleigh length is derived by the smaller beam waist. Under those conditions, confocal parameter is $2.93 \mu \mathrm{m}, N A_{x}=0.23$, and the aspect ratio is 2.4 , for plane wave; and confocal parameter is $4.94 \mu \mathrm{m}$, $N A_{x}=0.17$, and the aspect ratio is 3.3 for Gaussian beam, and confocal parameter is $4.94, N A_{x}=0.14$, and the aspect ratio is 4.0 under the third condition. Our experimental results are the range of 7.5 to 10 . So it shows that plane wave is the poorest and the condition of Gaussian beam in y direction and plane wave in $X$ direction is the most close to experiment.

When considering diffraction effects, we assume there are two independent beams with different beam waists going through a same objective. Gaussian laser beam is focused by a lens and the point of the absolute maximum irradiance of the focused field is located on the axis but at a distance difference $\Delta f$, as called focal shift [20-22]. An explicit expression of the relative focal shift in focused, apertured Gaussian beam:

$$
\begin{gathered}
\Delta f=\Delta f_{\mathrm{G}}\left[1-\exp \left(-0.3 \alpha^{2}\right)\right] \\
+\Delta f_{\mathrm{D}}\left[\exp \left(-0.3 \alpha^{2} / G\right)\right],
\end{gathered}
$$

where $\Delta f_{\mathrm{G}}=-f /\left(1+\pi^{2} G^{2}\right), G=\omega^{2} / \lambda f, f$ is focal length, $\lambda$ is the wavelength of laser. $\Delta f_{\mathrm{D}}=-f /\{1+N[1+$ $\left.\left.\left(\pi^{2} N / 12\right)^{1.51}\right]^{1 / 1.51}\right\}, N=\alpha^{2} / \lambda f, \alpha=N / G$, a is radius of circular aperture. $N$ is Fresnel number of diffracting aperture, represents the number of Fresnel zones that fill the aperture when the aperture is viewed from the geometrical focus. In our up mentioned parameters, Fresnel number is 704 in $y$ direction when laser beam is $3 \mathrm{~mm}$ (equal to the diameter of objective pupil), and focal shift is $8 \mathrm{~nm}$. However in $x$ direction Fresnel number is 12.5 when slit width is $0.4 \mathrm{~mm}$, it contributes a focal shift of $30.3 \mu \mathrm{m}$ which is bigger than confocal length (about $5 \mu \mathrm{m}$ ). Now it is clear that the foci in $x$ direction and in $y$ direction are not same position. Confocal parameter is extended extensively, can't calculated by Geometry optics. This situation is shown schematically in Fig. 1a. The effective focus of astigmatic beam should be located in the between $Z_{X 0}$ and $Z_{Y 0}$. By use formula (1), we calculated the dependence of focal shift on slits width, shown in Fig. 1b. Focal shift increases exponentially as the width of slit 


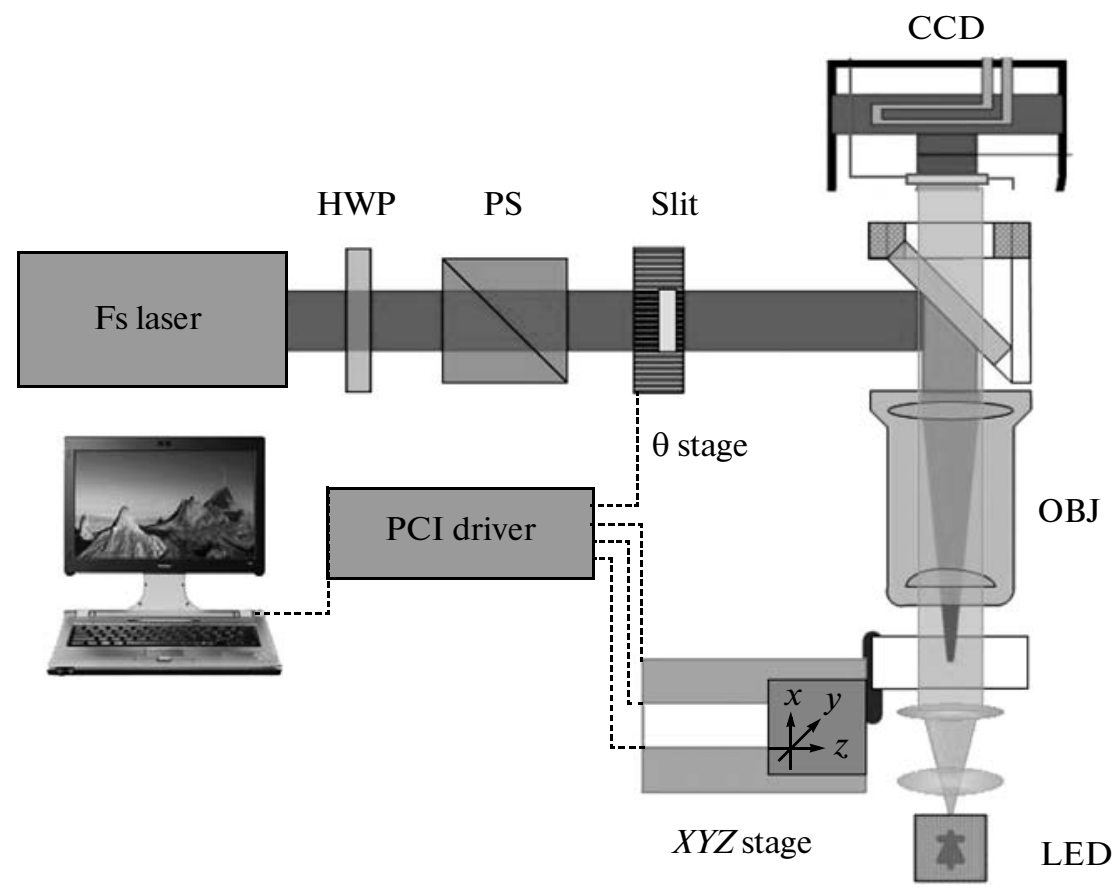

Fig. 2. Experimental setup of the femtosecond laser waveguide writing arrangement indicating the irradiation geometry: HWP half wave plate, PS polarized splitter, OBJ microscope objectives, LED light-emitting diode. The slit is fixed on a $Y Z$ stages which is in the holder of computer-controlled rotation stage. Another $Y Z$ stages under the rotation stage is used to align laser beam going through the center of the rotation stage.

decreases. It is easy to understand that smaller slit produces stronger diffraction. However it is difficult to calculate precise results based on Fraunhofer diffraction theory $[23,24]$.

\section{EXPERIMENT SETUP}

The structures presented below were achieved in the transversal configuration, where the sample is translated perpendicular to the laser propagation axis. Polished fused silica parallelepipedic samples are irradiated with 120 fs pulses from an $1 \mathrm{kHz}$ Ti:sapphire ultrafast regenerative amplifier laser systems (Spitfire, Spectra-Physics) which delivers an average power of $800 \mathrm{~mW}$ at the center wavelength of $800 \mathrm{~nm}$. A computer driven electromechanical shutter, synchronized with the movements of the positioning system (Physik Instrumente M-405.DG, M-126.DG, M-111.PD) permits the writing of longitudinal structures in the bulk of the sample. A long working distance microscope objective (Mitutoyo, NA $=0.55, \mathrm{WD}=13 \mathrm{~mm}$ ) is employed to focus the femtosecond beam in the silica glass. A Zernike-type positive optical phase-contrast microscopy (PCM) system is employed to monitor the laser-irradiated areas. The slits with sizes of $6.0 \times 0.5 \mathrm{~mm}, 6.0 \times 0.4 \mathrm{~mm}$, and $6.0 \times 0.3 \mathrm{~mm}$ are used to generate an astigmatic writing beam. The slit is in the holder of a rotation stage (Physik Instrumente M-060.DG). There are two $Y Z$ stages to help optical alignment. One stage makes sure that the writing laser beam goes through the center of the rotation stage, and another one to adjust the position of the slit at the center of the rotation stage. The movement of the rotation stage is decided by the curve of the designed waveguide. Since the effect of spherical aberration is not negligible thus leading to an increase of aspect ratio [16]. The experiment was conducted at a focal depth of $200 \mu \mathrm{m}$. The schematic of experimental setup is shown in Fig. 2.

\section{STRAIGHT WAVEGUIDE WRITING}

In order to determine the optimum slit width for fabricating waveguides with circular cross-sections, groups of channels were written at the same pulse energy but with slit widths ranging from $300 \mu \mathrm{m}$ up to $500 \mu \mathrm{m}$. After irradiation, the sample was side-polished and checked under an optical microscope in transmission mode. The aspect ratio near 1:1 obtained with 400 and $300 \mu \mathrm{m}$ slit. Looking back the focal shift shown in Fig. 1, it is easy to understand why there is big error between geometrical optics prediction and experimental results since the focal shift is more bigger than confocal parameter.

In relatively gentle exposure conditions, two intensity dependent regimes of positive refractive index modifications, hereafter referred to as type I and type II, were mentioned upon irradiation with $800 \mathrm{~nm}$ femtosecond laser pulses in early paper $[25,26]$. The guiding properties of type I and type II waveguides have 


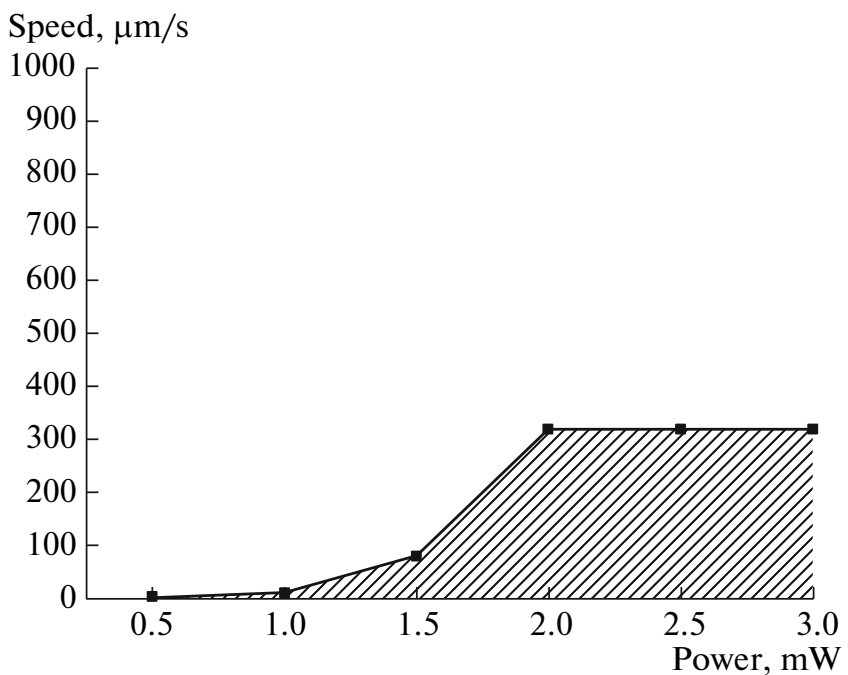

Fig. 3. Processing windows for generating type I transverse waveguiding traces in fused silica with a $400 \mu \mathrm{m}$ slit.

been analyzed in our recent paper [27]. The fantastical nonlinear effects $[5,28]$ such as supercontinuum generation in nanoporous doped glass in the structure induced by femtosecond laser is absent in our case. In transversal writing configuration, there provides type I and type II waveguides. And a schematic description of the processing window for each resulting waveguide type in a transversal writing configuration with the slit was given in Fig. 3, under following irradiation conditions: 100 fs pulse duration, radiation wavelength $800 \mathrm{~nm}$, laser repetition rate $1 \mathrm{kHz}$. The size of the slit is $6.0 \times 0.4 \mathrm{~mm}$, the NA of the focusing objective is $0.55 \mathrm{NA}$, the pupil of the objective is $3 \mathrm{~mm}$. The evaluation is performed at a working depth centered around $200 \mu \mathrm{m}$. The reported pulse length values were measured after the focusing objective and were determined by adjusting the compressor position to compensate additional dispersion. Region I produces smooth and low loss type I waveguide, and region 2 is according to type II waveguide which is high optical loss and polarization sensitive normally.

Figure 4 shows the phase contrast pictures and the cross-sections of the waveguides written at different speeds. Black cross-sections comes from the scattering or absorption of the waveguides, the resulting phase contrast pictures of the waveguides are white and possesses birefringence. When the scanning speeds is beyond $40 \mu \mathrm{m} / \mathrm{s}$, the cross-section is white in transmission microscopy, which shows a good guiding property. Figure 5 shows the cross-section of the waveguides written with $300 \mu \mathrm{m}$ slit at different scanning speed. The diameter of the type I waveguides almost keeps constant under different scanning speeds, is about $5.2 \mu \mathrm{m}$, this value is very close calculated one when taking half maximum full width (HMFW) of a Gaussian beam waist (in our case, it is

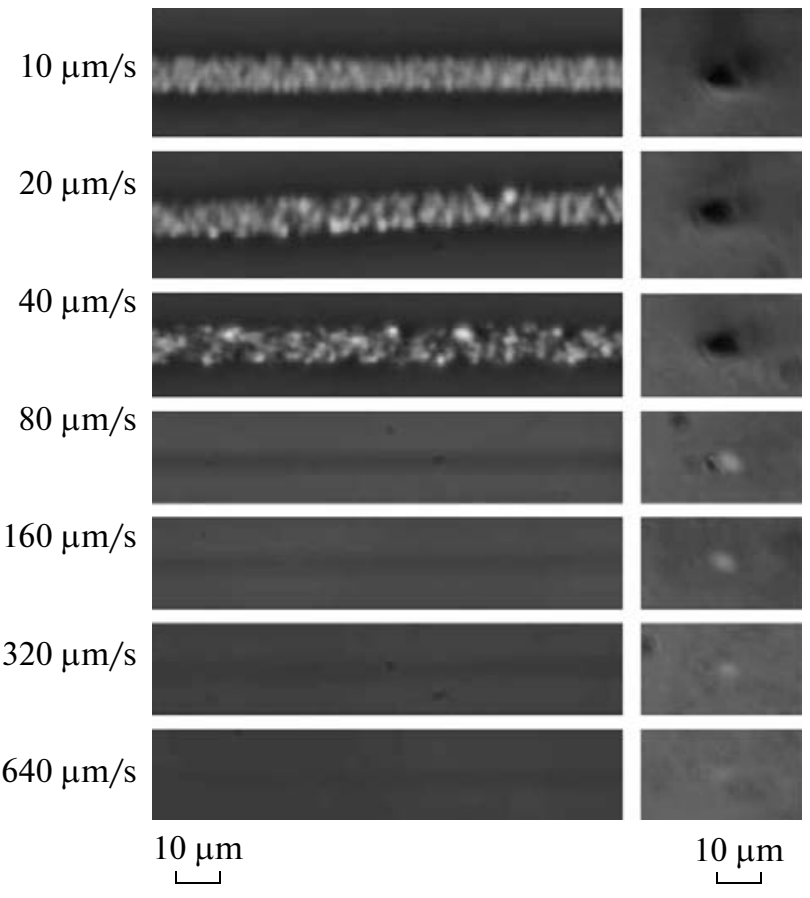

Fig. 4. Transversal waveguides and cross-section in $\mathrm{SiO}_{2}$ written by $800 \mathrm{~nm}$ femtosecond laser radiation at different scanning speeds. The width of the slit is $400 \mu \mathrm{m}$. Images in the left frame show top view PCM pictures of photowritten waveguides at different speeds indicated on the left side. The dark colors indicate a positive refractive index change and the white colors suggest negative index variations or light scattering. The right frame shows the cross-sections under transmission microscopy. The white spots show the collecting ability of the waveguides, inverse the black spots show strong scattering or absorption. The power is $1.5 \mathrm{~mW}$ after the slit.

4.94). The guiding properties were checked by injection $\mathrm{HeNe}$ laser, it is a typical type I waveguide, and there is no polarization effect and birefringence.

\section{CURVED WAVEGUIDE WRITING}

Based on straight waveguide writing condition, we conducted $90^{\circ}$ circular arc waveguide writing. As the rule of thumb, realization of circular cross-section arc waveguide fabrication requires a rotating slit. The long axis of the slit keeps parallel to the tangent line of the waveguide arc. Circular arc simplifies this calculation requirement of tangent line of curve. During writing, just keep the angle of slit equal to that of the writing arc. The inset rectangles in Fig. 6a present the direction of the slit while writing an arc. Femtosecond laser inscribed waveguides feature very low index changes and always qualify as weakly guiding structures in fused silica. Typically, index changes is between 0.0001 and 0.001 in silica $[4,29]$. Considering this small refractive index changes leads to huge bend loss at small radius, we wrote an arc with a radius of 500 and $600 \mu \mathrm{m}$. A 
(a)

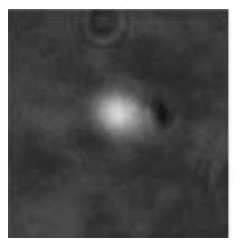

(b)

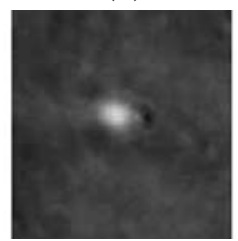

(c)

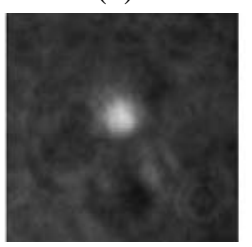

(d)

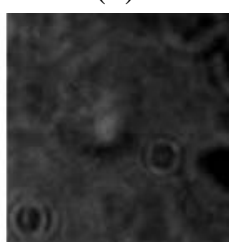

Fig. 5. The cross-sections of the waveguides written by $800 \mathrm{~nm}$ femtosecond laser radiation at different scanning speeds. The width of the slit is $300 \mu \mathrm{m}$. the writing power is $2 \mathrm{~mW}$ after the slit. The scanning speeds in (a) $-(\mathrm{d})$ are 20 , 40 , 80 , and $160 \mu \mathrm{m} / \mathrm{s}$, respectively.

square fused silica sample is employed for convenient mode check of waveguide two cross-sections at two ends. The laser power is $1.2 \mathrm{~mW}$. Linear velocity is about $50 \mu \mathrm{m} / \mathrm{s}$ here.

Figures $6 \mathrm{~b}$ and $6 \mathrm{c}$ are the cross sections of the two ends of the waveguide. When we check out the crosssections of the arc waveguides, the sample is rotated to vertical in compared with the writing condition. So there is a shadow in the cross-section pictures. Figure $6 \mathrm{~d}$ is a portion of the arc waveguide viewing in transmission microscopy. (a)

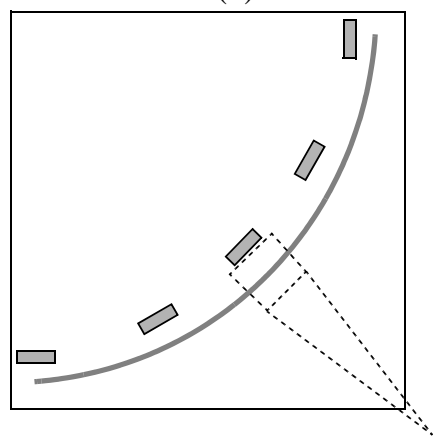

(c)

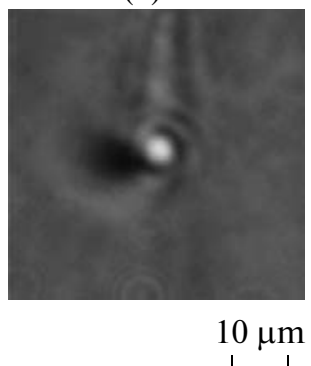

(b)

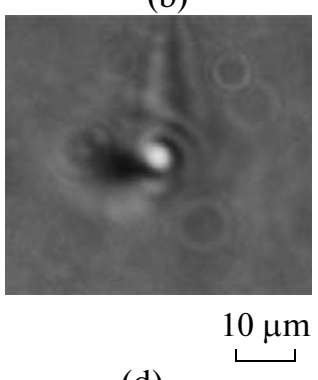

(d)

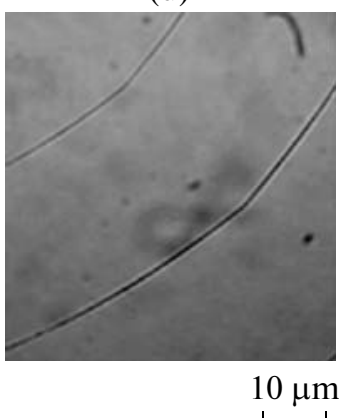

Fig. 6. A $90^{\circ}$ arc waveguide and its two end cross-section modes, (a) is schematic view of the photoinscribed arc structure in fused silica using ultrashort pulsed laser radiation, the inset rectangles shows the directions of the slit. $(b, c)$ Cross sections of the two ends of the waveguide of the $600 \mu \mathrm{m}$ arc. (d) A portion of the arc waveguide viewing in transmission microscopy. The radius of the arc are 500 and $600 \mu \mathrm{m}$ in (d), respectively. The writing power is $1.2 \mathrm{~mW}$.

\section{CONCLUSIONS}

We demonstrated how to realize a fabrication of circular cross-section waveguide in two dimensions with a dynamical slit. It is helpful to planer light-wave circuit (PLC) chip in glass. The dependence of crosssection on width of the slit was investigated in experiment, and processing window for type I waveguide was given under the conditions of our parameters. The difference of slit width between geometrical optics prediction and experimental result was explained by considering the focal shift in $x y$ directions due to diffraction effects.

\section{ACKNOWLEDGMENTS}

This work was supposed by West Light Foundation of The Chinese Academy of Sciences (no. 0729591213) and Innovative Research International Partnership Project of The Chinese Academy of Sciences

\section{REFERENCES}

1. R. R. Gattass and E. Mazur, Nature Photon. 2, 219 (2008).

2. G. Della Valle, R. Osellame, and P. Laporta, J. Opt. A: Pure Appl. Opt. 11, 13001 (2009).

3. M. Ams, G. Marshall, P. Dekker, J. Piper, and M. Withford, Laser Photon. Rev. 2, 1 (2008).

4. A. M. Zheltikov, Laser Phys. Lett. 1, 220 (2004).

5. V. N. Bagratashvili, E. A. Chutko, V. M. Gordienko, I. A. Makarov, and M. A. Timofeev, Laser Phys. Lett. 5, 671 (2008).

6. C. Mauclair, A. Mermillod-Blondin, N. Huot, E. Audouard, and R. Stoian, Opt. Express 16, 5481 (2008).

7. G. Cerullo, R. Osellame, S. Taccheo, M. Marangoni, D. Polli, R. Ramponi, P. Laporta, and S. De Silvestri, Opt. Lett. 27, 1938 (2002).

8. Y. Cheng, K. Sugioka, K. Midorikawa, M. Masuda, K. Toyoda, M. Kawachi, and K. Shihoyama, Opt. Lett. 28, 55 (2003).

9. R. Osellame, S. Taccheo, M. Marangoni, R. Ramponi, P. Laporta, D. Polli, S. De Silvestri, and G. Cerullo, J. Opt. Soc. Am. B 20, 1559 (2003). 
10. N. D. Psaila, R. R. Thomson, H. T. Bookey, A. K. Kar, N. Chiodo, R. Osellame, G. Cerullo, G. Brown, A. Jha, and S. Shen, Opt. Express 14, 10452 (2006).

11. M. Ams, G. D. Marshall, D. J. Spence, and M. J. Withford, Opt. Express 13, 5676 (2005).

12. S. Sowa, W. Watanabe, T. Tamaki, J. Nishii, and K. Itoh, Opt. Express 14, 291 (2006).

13. V. Diez-BIanco, J. Siegel, A. Ferrer, A. Ruiz de la Cruz, and J. Solis, Appl. Phys. Lett. 91, 051104 (2007).

14. R. R. Thomson, A. S. Bockelt, E. Ramsay, S. Beecher, A. H. Greenaway, A. K. Kar, and D. T. Reid, Opt. Express 16, 12786 (2008).

15. Yusuke Nasu, Masaki Kohtoku, and Yoshinori Hibino, Opt. Lett. 30, 723 (2005).

16. K. J. Moh, Y. Y. Tan, X. C. Yuan, D. K. Y. Low, and Z. L. Li, Opt. Express 13, 7288 (2005).

17. W. Yang, C. Corbari, P. G. Kazansky, K. Sakaguchi, and I. C. S. Carvalho, Opt. Express 16, 16215 (2008).

18. N. Nguyen, A. Saliminia, S. Chin, and R. Vallee, Appl. Phys. B 85, 145 (2006).
19. www.mellesgriot.com/products/optics/gb_2_3.htm

20. H. Osterberg and L. W. Smith, J. Opt. Soc. Am. 51, 1050 (1961).

21. Y. Li, J. Mod. Opt. 39, 1761 (1992).

22. W. H. Carter, Appl. Opt. 21, 1989 (1982).

23. Yajun Li, J. Opt. Soc. Am. A 25, 1835 (2008).

24. Xinyue Du and Dapmu Zhao, Appl. Opt. 45, 9049 (2006).

25. Y. Shimotsuma, P. G. Kazansky, J. Qiu, and K. Hirao, Phys. Rev. Lett. 91, 247405/1-4 (2003).

26. V. R. Bhardwaj, E. Simova, P. P. Rajeev, C. Hnatovsky, R. S. Taylor, D. M. Rayner, and P. B. Corkum, Phys. Rev. Lett. 96, 057404/1-4 (2006).

27. G. Cheng, K. Mishchik, C. Mauclair, E. Audouard, and R. Stoian, Opt. Express 17, 9515 (2009).

28. A. M. Zheltikov and D. T. Reid, Laser Phys. Lett. 5, 11 (2008).

29. C. Mauclair, G. Cheng, N. Huot, E. Audouard, A. Rosenfeld, I. V. Hertel, and R. Stoian, Opt. Express 17, 3531 (2009). 